\title{
Modelación del perfil fustal y volumen total para Pinus ayacahuite Ehren
}

\section{Taper and total volume model for Pinus ayacahuite Ehren}

\author{
Agustín Ramírez-Martínez', Wenceslao Santiago-García2*, Gerónimo Quiñonez-Barraza, \\ Faustino Ruiz-Aquino² y Pablo Antúnez ${ }^{2}$
} ' Universidad de la Sierra Juárez. Ingeniería Forestal.
Ixtlán de Juárez, Oaxaca, México.

\author{
2 Universidad de la Sierra Juárez. Instituto de Estudios \\ Ambientales-División de Estudios de Postgrado. \\ Oaxaca, México.
}

* Autor de correspondencia.wsantiago@unsij.edu.mx

\begin{abstract}
3 Instituto Nacional de Investigaciones Forestales Agrícolas y Pecuarias. Centro de Investigación Regional Norte Centro. Campo Experimental Valle del Guadiana. Durango, Durango, México.
\end{abstract}

\section{RESUMEN}

El perfil del fuste o ahusamiento describe el cambio del diámetro conforme aumenta la altura del árbol a partir del tocón. Para describir esta variación y cuantificar las secciones del fuste de árboles en pie, se han utilizado modelos matemáticos, los cuales constituyen una herramienta estadística flexible para procesar datos y determinar existencias y productos maderables. El objetivo de esta investigación fue modelar el ahusamiento de Pinus ayacahuite Ehren que se distribuye en rodales mezclados bajo manejo forestal, en la región Sierra Norte de Oaxaca. Con datos de 55 individuos de la especie estudiada se ensayaron cinco modelos de ahusamiento cuyos ajustes fueron por regresión lineal y no lineal. La evaluación y selección de los modelos más apropiados se hizo con base en indicadores estadísticos de bondad de ajuste, además de un análisis gráfico. Dada la capacidad predictiva del modelo Cielito 2, este resultó ser el más adecuado para describir el perfil del fuste y determinar la distribución de productos maderables a un diámetro de punta y altura deseados. Al evaluar la estimación del volumen a través de diferentes alternativas, la más adecuada resultó ser mediante una ecuación de volumen de doble entrada, debido a que presentó el menor sesgo en la estimación. Aunque la ecuación de volumen total definida en la integración del modelo Cielito 2 posee la capacidad de estimar el volumen entre dos alturas a lo largo del fuste, la utilidad mayor es para la distribución de productos de árboles en pie.

PALABRAS CLAVE: ahusamiento, ajuste de modelos, distribución de productos, volumen total.

\section{ABSTRACT}

The stem profile or taper describes the change in diameter against the increasing height of the tree. In order to describe this variation and quantify bole volume sections of standing trees, mathematical models commonly have been used, because these statistical tools provide a flexible representation to estimate stocks and forest products classification. The objective of this research was to model the stem profile of Pinus ayacabuite Ehren fitting 5 taper models by linear and non-linear least squares methods using data of 55 trees of $P$. ayacahuite. The evaluation and selection of the best model was based on statistical indicators and a graphical analysis. The Cielito 2 model best described the stem taper of $P$. ayacabuite and was superior for estimating the distribution of timber products. Evaluating different ways to estimate the stem volume, the most appropriate model considers using the volume equation, because it showed a lower bias; although the integration of Cielito 2 model has the capacity to estimate the volume between two heights along the stem, it is more useful for estimating products classification of standing trees.

KEYWORDS: taper, model fitting, forest products classification, total volume.

\section{INTRODUCCIÓN}

La forma de un árbol es compleja de representar porque presenta cambios a lo largo del fuste: en la base tiene la forma de un neiloide, en el centro un paraboloide o cilindro y en la parte superior un cono (Avery y Burkhart, 2001); por lo general, el perfil del fuste de un árbol es monopódico o de tipo excurrente, típicamente en coníferas (Burkhart y Tomé, 2012).
La estimación directa del volumen de árboles individuales es crucial en inventarios forestales; por esto, es necesario contar con expresiones matemáticas que permitan modelar el ahusamiento y estimar los volúmenes total o parcial del fuste, mediante variables de fácil medición.

El perfil del fuste o ahusamiento de un árbol se define como el coeficiente de estrechamiento del diámetro con el incremento 
en altura; por tanto, las funciones de ahusamiento o perfil describen los diámetros esperados, con o sin corteza, a distintas alturas en el fuste. La variación del ahusamiento en el fuste del árbol es causada por las condiciones naturales de crecimiento, y por la respuesta del bosque a los tratamientos silvícolas aplicados (Hernández-Pérez, De los Santos-Posadas, Ángeles-Pérez, Valdéz-Lazalde y Volke-Haller, 2013; Uranga-Valencia, De los Santos-Posadas, Valdez-Lazalde, López-Upton y Navarro-Garza, 2015). El ahusamiento puede ser descrito mediante una función matemática que permita predecir los diámetros a diferentes alturas del fuste; predecir la altura para un determinado diámetro fustal y predecir el volumen total del fuste o entre distintas alturas fustales mediante la integración del modelo (Davel y Trincado, 2000; Kozak, 2004; Pompa-García, Corral-Rivas, HernándezDíaz y Álvarez-González, 2009a; Pompa-García, Hernández, Prieto-Ruiz y Dávalos-Sotelo, 2009b; López, Barrios y Trincado, 2015).

El supuesto sobre el que se basan estas funciones es que la forma del árbol varía continuamente a lo largo de la longitud del fuste y es monotónicamente decreciente a medida que la distancia del tocón a la punta comercial se aproxima a la altura total (Lee et al., 2003). Los modelos de ahusamiento constituyen una herramienta importante para determinar existencias maderables (al integrar la ecuación de ahusamiento entre dos alturas) y realizar la clasificación de productos de árboles en pie.

Existen varios modelos que han sido utilizados para describir el perfil fustal, y se han clasificado de acuerdo con la complejidad de las expresiones matemáticas, el número de variables y los coeficientes del modelo; la clasificación general se basa en: modelos generales para árbol individual, modelos segmentados y modelos de exponente variable (Burkhart y Tomé, 2012).

Durante el desarrollo de los primeros estudios se utilizaron modelos polinomiales con diferente grado (Bruce, Curtis y Van Coevering, 1968; Omerod, 1973; Real y Moore, 1986). Otros han empleado funciones polinomiales segmentadas (Bruce et al., 1968; Max y Burkhart, 1976; Cao, Burkhart y Max, 1980) y en algunos más, funciones geométricas y trigonométricas (Parresol y Thomas, 1996; Fang y Bailey, 1999; Bi, 2000).

Con la integración de la función de ahusamiento, evaluada entre dos alturas, se obtiene una expresión con la capacidad de estimar el volumen comercial (Demaerschalk, 1972). Pérez, Burkhart y Stiff (1990) señalan que las funciones de ahusamiento son útiles cuando los árboles se utilizan para diferentes fines en la industria forestal.

En la región de la Sierra Norte de Oaxaca, Pinus ayacabuite Ehren es una de las especies con mayor importancia comercial, por esto fue necesario desarrollar modelos para determinar el tipo y volumen de productos a obtener en las intervenciones forestales.

\section{OBJETIVOS}

Modelar el ahusamiento y el volumen fuste total de Pinus ayacabuite Ehren en los bosques de Ixtlán de Juárez, Oaxaca, México.

\section{MATERIALES Y MÉTODOS}

\section{Área de estudio}

La comunidad de Ixtlán de Juárez, Oaxaca, se ubica entre las coordenadas $17^{\circ} 18^{\prime} 16^{\prime \prime}$ y $17^{\circ} 34^{\prime} 00^{\prime}$ ' de latitud norte y $96^{\circ} 20^{\prime}$ 00" y 96० 31'38" de longitud oeste. Los tipos de climas predominantes en el área son templado subhúmedo y templado subhúmedo con lluvias en verano, con temperatura promedio anual de $20^{\circ} \mathrm{C}$ (Castellanos-Bolaños, Treviño-Garza, AguirreCalderón, Jiménez-Pérez y Velázquez-Martínez, 2010). Los tipos de suelo más comunes son: acrisol, luvisol y cambisol. El principal tipo de vegetación en el área son los bosques de pino-encino (Ruiz-Aquino et al., 2014).

\section{Datos dasométricos}

En este estudio se utilizaron 844 pares de datos de diámetro con corteza y altura, los cuales se obtuvieron de 55 árboles de $P$. ayacahuite distribuidos en un intervalo amplio de categorías de diámetro y altura (Tabla 1). Cada árbol fue derribado y seccionado, efectuándose mediciones del diámetro normal $(D)$, diámetro del tocón $(d b)$, así como de los diámetros con corteza $(\mathrm{dm})$ y alturas $(\mathrm{hm})$ tomadas a diferentes secciones a partir del tocón $(b b)$ hasta abarcar la altura total del árbol $(H)$.

El resumen de las estadísticas descriptivas de los análisis troncales se presenta en la tabla 1. La dispersión de las alturas $(\mathrm{hm} / \mathrm{H})$ y diámetros $(\mathrm{dm} / \mathrm{D})$ en términos relativos se ilustra en la figura 1. 
TABLA 1. Valores estadísticos descriptivos de los árboles estudiados.

\begin{tabular}{llllll}
\hline \multirow{2}{*}{ Variable } & $N$ & Media & Desviación estándar & Mínimo & Máximo \\
\hline$D$ & 55 & 39.58 & 13.34 & 9.40 & 71.00 \\
$d b$ & 55 & 50.95 & 15.40 & 13.40 & 86.00 \\
$h b$ & 55 & 0.18 & 0.05 & 0.06 & 0.38 \\
$H$ & 55 & 27.25 & 4.37 & 9.87 & 33.37 \\
$d m$ & 844 & 26.16 & 16.76 & 0.001 & 86.00 \\
$h m$ & 844 & 12.24 & 9.61 & 0.09 & 33.37 \\
\hline
\end{tabular}

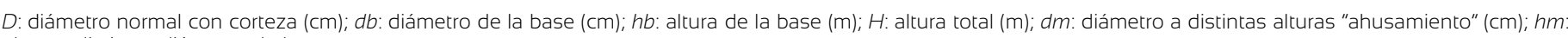
altura a distintos diámetros $(\mathrm{m})$.

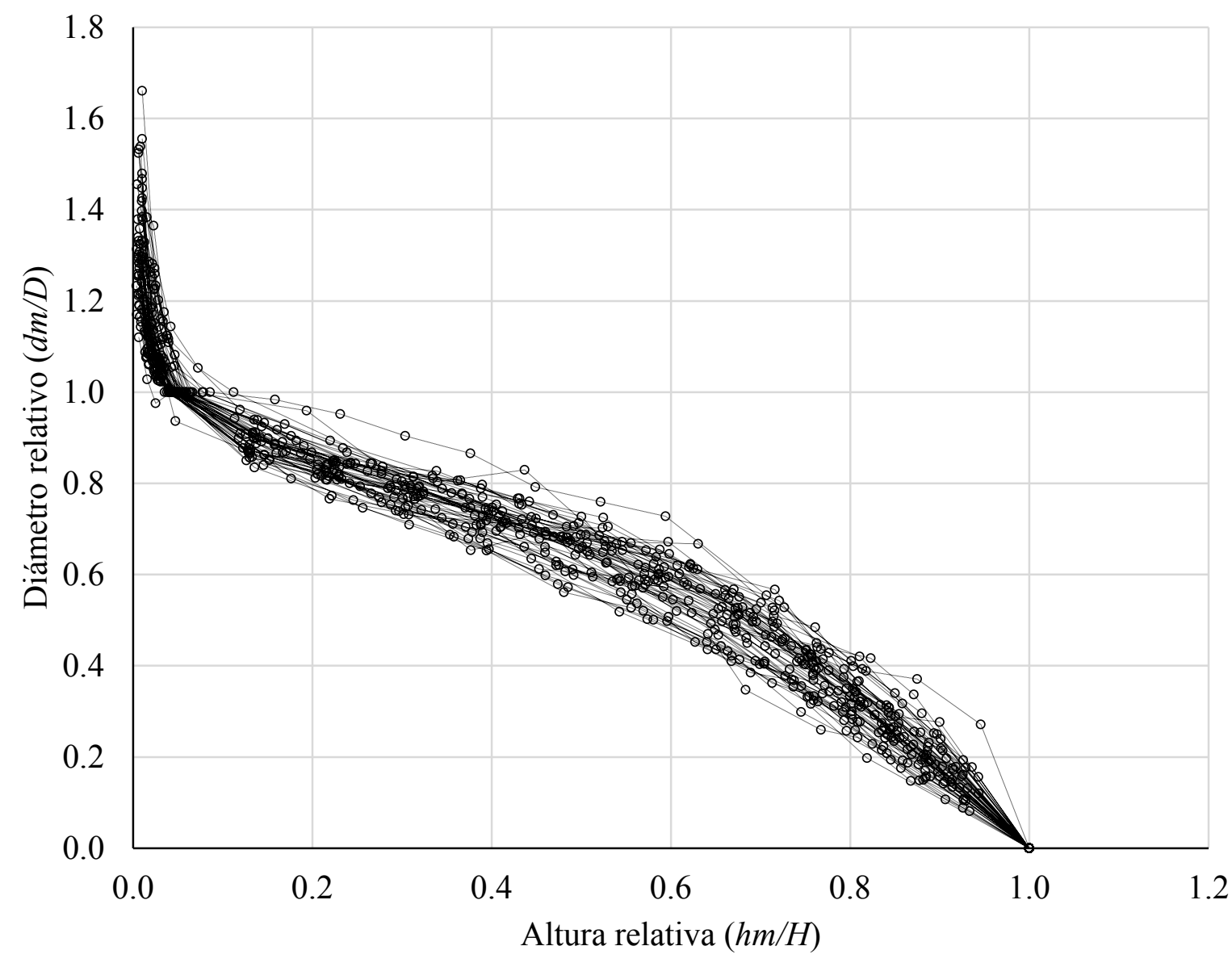

FIGURA 1. Dispersión del diámetro relativo y la altura relativa 


\section{Modelos de ahusamiento seleccionados}

Los modelos de ahusamiento utilizados para describir el perfil del fuste de $P$. ayacabuite fueron agrupados en la categoría de modelos fustales generales para árbol individual (modelos polinómicos simples y de tipo exponencial); la mayoría fueron desarrollados y aplicados para especies de coníferas. Todas las ecuaciones se expresaron en términos del diámetro $(\mathrm{dm})$ como variable dependiente (Tabla 2), para evitar los errores multiplicativos que se presentan cuando se usa el diámetro relativo (Honorato, 2011); además, el diámetro permite realizar comparaciones directas de bondad de ajuste y capacidad predictiva entre los modelos.

\section{Procedimiento de ajuste}

En el análisis estadístico se utilizaron los 844 pares de datos para estimar los parámetros de los modelos de ahusamiento, con regresión lineal y no lineal, a través del procedimiento "MODEL" del paquete SAS/ETS® (SAS Institute Inc., 2011).
Los criterios para calificar la bondad de ajuste se basaron en métodos analíticos (Tabla 3); además, se consideró el número de parámetros empleados en la estructura (a menor número de parámetros mayor parsimonia). Todos estos criterios se evaluaron en un sistema de calificación relativa, asignándole un valor de acuerdo con el orden de importancia (del 1 al 5 siendo 1 el mejor calificado y 5 el peor). La sumatoria de los valores conformó la calificación final (CF) y mediante una comparación se identificó al modelo mejor, siendo aquel que presentó la calificación total más baja, así como una distribución de los residuales más homogénea.

Además del análisis gráfico y estadístico (con los estadísticos de ajuste), se evaluó la significancia de los estimadores de los parámetros, a través de pruebas de hipótesis con la distribución t Student, bajo la hipótesis nula H0: $\boldsymbol{\beta} \mathbf{i}=0$ y Ha: $\boldsymbol{\beta} \mathbf{i} \neq 0$ (Di Rienzo et al., 2005), para esto se utilizó un valor crítico de $\alpha=0.05$.

TABLA 2. Modelos de ahusamiento ajustados a $P$. ayacabuite.

Modelo Expresión Nombre

$1 d m=D \times\left[\beta_{1}\left(\frac{H-h m}{H}\right)+\beta_{2}\left(\frac{H-h m}{H}\right)^{2}+\beta_{3}\left(\frac{H-h m}{H}\right)^{3}\right]^{1 / 2}+\varepsilon \quad \begin{aligned} & \text { Cielito } 1 \text { (Rentería, Ramírez y } \\ & \text { Zamudio, 2006) }\end{aligned}$

$2 d m=D \times\left[\beta_{0}+\beta_{1}\left(\frac{h m}{H}\right)+\beta_{2}\left(\frac{h m}{H}\right)^{2}+\beta_{3}\left(\frac{h m}{H}\right)^{3}+\beta_{4}\left(\frac{h m}{H}\right)^{4}\right]^{1 / 2}+\varepsilon$

Cielito 2 (Rentería et al., 2006)

$3 \quad d m=D \times\left[\beta_{1}\left(\frac{H-h m}{H}\right)+\beta_{2}\left(\frac{H-h m}{H}\right)^{\beta_{3}}\right]^{1 / 2}+\varepsilon$

Cielito 3 (Rentería et al., 2006)

4

$$
d m=\beta_{0} D^{\beta_{1}} H^{\beta_{2}}(H-h m)^{\beta_{3}}+\varepsilon
$$

Clutter (Clutter,1980)

5

$$
d m=D\left(\beta_{1}+\beta_{2} \ln \left(1-\left(1-e^{\frac{-\beta_{1}}{\beta_{2}}}\right)\left(\frac{h m}{H}\right)^{\frac{1}{3}}\right)\right)+\varepsilon
$$


TABLA 3. Indicadores estadísticos utilizados para evaluar la bondad de ajuste de los modelos de ahusamiento.

\begin{tabular}{lc}
\hline Estadístico & Ecuación \\
\hline Coeficiente de determinación ajustado (Rªdj) & $R_{a d j}^{2}=1-\frac{(n-1) \sum_{i=1}^{n}\left(y_{i}-\hat{y}_{i}\right)^{2}}{(n-p) \sum_{i=1}^{n}\left(y_{i}-\bar{y}_{i}\right)^{2}}$ \\
Raíz del cuadrado medio del error (REMC) & $R E M C=\sqrt{\frac{\sum_{i=1}^{n}\left(y_{i}-\hat{y}_{i}\right)^{2}}{n-p}}$ \\
Sesgo promedio $(\bar{E})$ & $\bar{E}=\frac{\sum_{i=1}^{n}\left(y_{i}-\hat{y}_{i}\right)}{n}$ \\
Criterio de información de Akaike (AIC) & $A I C=2 \mathrm{p}+\mathrm{n} \ln \left(\frac{\sum_{i=1}^{n}\left(y_{i}-\hat{y}_{i}\right)^{2}}{n}\right)$ \\
\hline
\end{tabular}

$y_{i}$ : valores observados; $\hat{y}_{i}$ : valores predichos; $\bar{y}$ : media de los valores observados; $p$ : número de parámetros del modelo; $n$ : número de observaciones.

\section{Verificación de los supuestos del modelo de regresión}

La normalidad en la distribución de los residuales se verificó con la prueba de Shapiro-Wilk (Shapiro y Wilk, 1965), al realizar la prueba de hipótesis siguiente: $H_{0}: \varepsilon \sim$ Normal, bajo la regla de decisión: si el valor $p<=\alpha$ entonces $H_{0}$ se rechaza, para esta prueba también se utilizó un valor de significancia del 5\% $(\alpha=$ 0.05).

Para probar la independencia de los residuales, se realizó la prueba de Durbin-Watson (DW) la cual mide el grado de autocorrelación de los residuos. Para este estadístico, el valor es aproximadamente igual a dos cuando los residuales son independientes; en caso contrario, si los residuales están correlacionados positivamente, tenderán a ser próximos a cero, o próximos a cuatro si los residuales están correlacionados negativamente (Durbin y Watson, 1971).

El supuesto de homogeneidad de varianzas se comprobó mediante la prueba de White (White, 1980). Para esto se efectuó la regresión de los residuales frente a todas las variables explicativas del modelo, los cuadrados y productos cruzados, bajo la hipótesis nula de varianza residual homogénea: $H_{0}: \sigma_{i}^{2}=\sigma^{2}$ para toda $i$. En muestras suficientemente grandes se puede comprobar que la variable definida como el producto entre el coeficiente de determinación de la regresión efectuada y el tamaño muestral $n$, seguirá una distribución $\chi^{2}$ - de White con $m$ grados de libertad, $n R^{2} \approx x_{m}^{2}$, donde $m$ denota el número de variables explicativas de la regresión realizada, cuando el valor $p$ obtenido fue menor que el nivel de significancia (valor $p<\alpha$ ) se rechazó la hipótesis nula a contrastar, y se tuvo un problema de heterocedasticidad (Díaz y Llorente, 2013).

\section{Comparación de estimaciones de volumen}

Para comparar el volumen del fuste total con corteza generado a partir de la integración del modelo de ahusamiento Cielito 2 (Anexo 1), se implementaron diferentes estrategias matemáticas (Tabla 4). En este caso, los modelos de volumen usados fueron: Schumacher-Hall de doble entrada y Berkhout de una entrada (Ramírez-Martínez, Santiago-García, Quiñonez-Barraza, RuizAquino y Martínez-Antúnez, 2016a) contra el volumen observado (obtenido mediante las fórmulas de Smalian y del cono); para esto, se seleccionaron 13 árboles, distribuidos en todas las categorías diamétricas de la base de datos.

\section{Resultados}

\section{Modelos de ahusamiento}

Los valores de los parámetros estimados para los modelos ajustados, el error estándar y el nivel de probabilidad se resumen en la tabla 5, para todos los modelos, los parámetros fueron significativos $(p<0.0201)$, al utilizar un valor crítico de nivel de significancia de $\alpha=0.05$.

Con respecto al error estándar de los parámetros, el modelo Cielito 2 (Modelo 2) presentó los valores relativamente más altos en los cinco parámetros de la estructura matemática. Por su parte, el modelo Biging (1984) (Modelo 5) presentó los errores más bajos en los estimadores de los parámetros. Sin embargo, 
deacuerdo con los criterios de bondad de ajuste y al sistema de calificación de escala relativa (Tabla 6), el modelo Cielito 2 presentó el ajuste mejor respecto a los otros modelos ajustados, al obtener la calificación final más baja (11 unidades), esto debido a que mostró el $R^{2}$ adj más alto, lo que indica que el modelo explica
97\% de la variabilidad total presente en la variable dependiente (diámetro), con un sesgo promedio $(\bar{E})$ de $0.26 \mathrm{~cm}$; además, mostró una precisión alta en las estimaciones del diámetro a lo largo del fuste, esto dado por sus valores menores en REMC y AIC.

TABLA 4. Expresiones matemáticas empleadas para la comparación del volumen fustal con corteza.

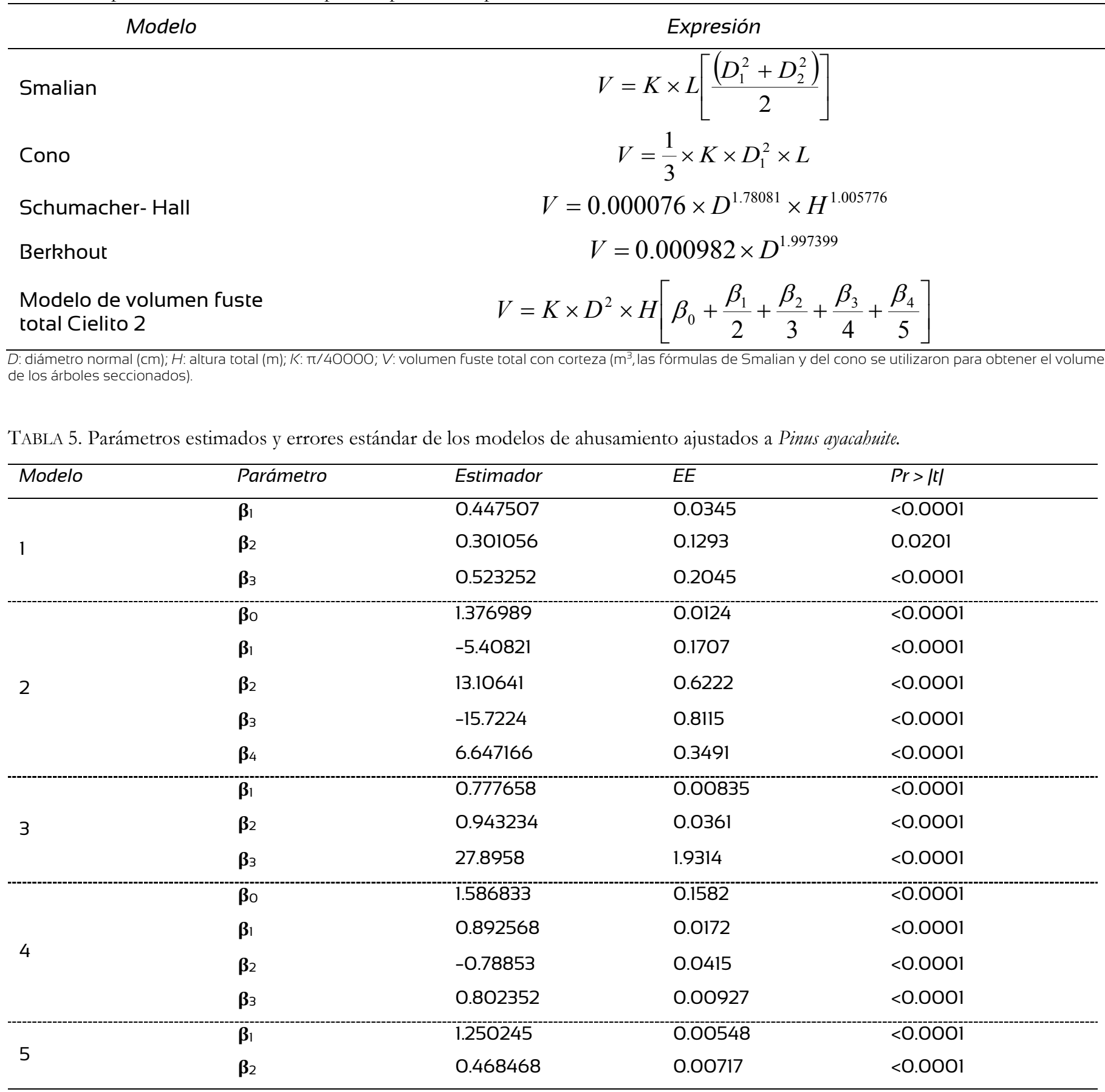

EE: error estándar; $\operatorname{Pr}>|||l|$ valor de la probabilidad de $t$ asociada al estimador del parámetro. 
TABLA 6. Estadísticos de bondad de ajuste de los cinco modelos de ahusamiento para describir el ahusamiento de Pinus ayacahuite Ehren.

\begin{tabular}{lllllll}
\hline Modelo & $n p$ & $R^{2} a d j$ & REMC & $\bar{E}$ & AIC & CF \\
\hline $\mathbf{1}$ & $3(2)$ & $0.96(5)$ & $3.19(5)$ & $0.23(3)$ & $1965.15(5)$ & 20 \\
$\mathbf{2}$ & $5(4)$ & $0.97(1)$ & $2.70(1)$ & $0.26(4)$ & $1683.19(1)$ & 11 \\
3 & $3(2)$ & $0.96(3)$ & $3.04(2)$ & $-0.13(2)$ & $1884.44(3)$ & 12 \\
4 & $4(3)$ & $0.96(4)$ & $3.16(4)$ & $0.07(1)$ & $1946.73(4)$ & 16 \\
5 & $2(1)$ & $0.97(2)$ & $2.71(2)$ & $0.31(5)$ & $1687.38(2)$ & 12 \\
\hline
\end{tabular}

np: número de parámetros del modelo; $R^{2}$ adj: coeficiente de determinación ajustado; $R E M C$ : raíz del cuadrado medio del error; E: sesgo promedio; AlC: criterio de información de Akaike; CF: calificación final.

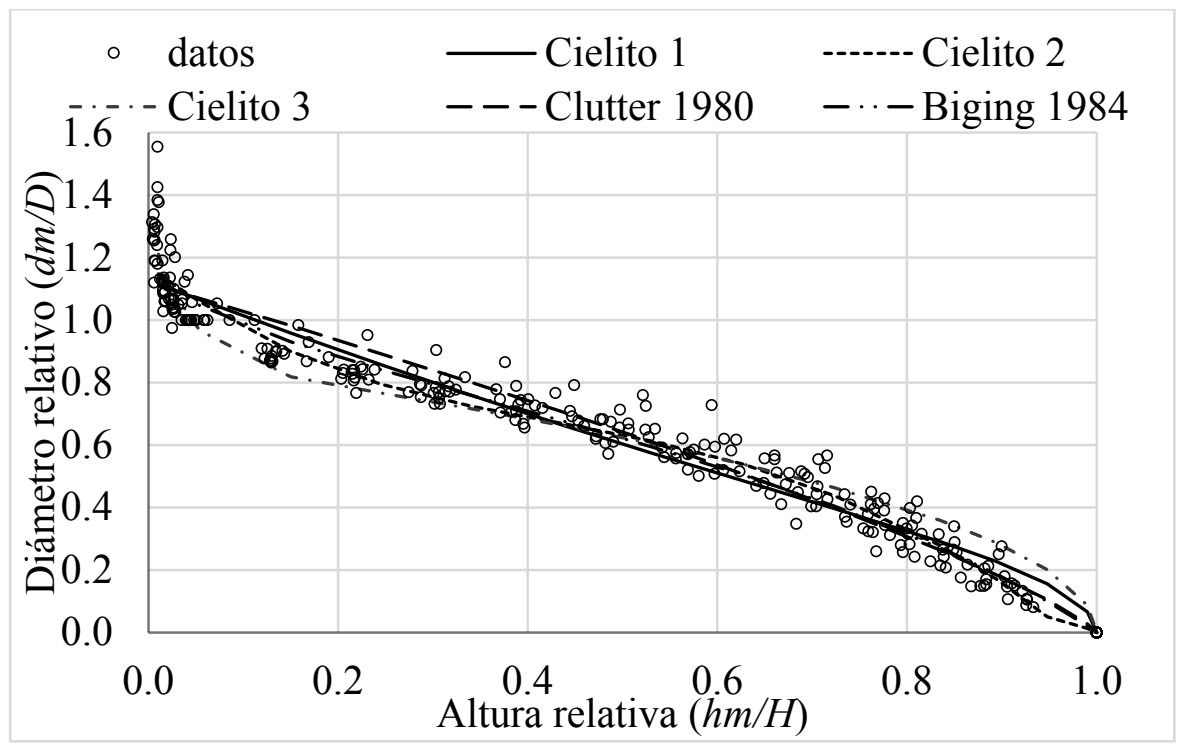

FIguRA 2. Comportamiento promedio de las cinco funciones de ahusamiento sobrepuestas a los datos de los perfiles fustales de $P$. ayacabuite.

La segunda mejor calificación la obtuvieron los modelos de Biging (1984) y Cielito 3, al explicar 97\% y 96\%, respectivamente, de la variabilidad del diámetro, y los valores de REMC y AIC fueron similares a los obtenidos por el modelo Cielito 2, aunque el sesgo promedio en la predicción del modelo de Biging (1984) fue más alto $(0.31 \mathrm{~cm})$ que el de Cielito 2 , en tanto que el modelo Cielito 3 presentó un sesgo menor absoluto con respecto a estos (0.13) (Tabla 6).

Aun cuando los modelos Cielito 2, Cielito 3 y Biging (1984) obtuvieron las puntuaciones más bajas, la discriminación final de estos se hizo a partir del análisis de la capacidad predictiva. Para esto se realizó una representación gráfica de los cinco modelos de ahusamiento al describir los diámetros $(d m)$ a diferentes alturas $(\mathrm{hm})$ a lo largo del fuste y de esta manera tener mayor certidumbre al seleccionar el modelo estadísticamente más preciso (Fig. 2).

$\mathrm{Al}$ considerar los valores de los estadísticos de bondad de ajuste y el análisis gráfico de los cinco modelos, el modelo Cielito 2 describió mejor el ahusamiento de P. ayacahuite. En la figura 3, se presentan los residuales contra los valores predichos por el modelo Cielito 2, se puede observar que existen datos en ambos lados de la línea de referencia (línea cero) manifestando un equilibrio. 


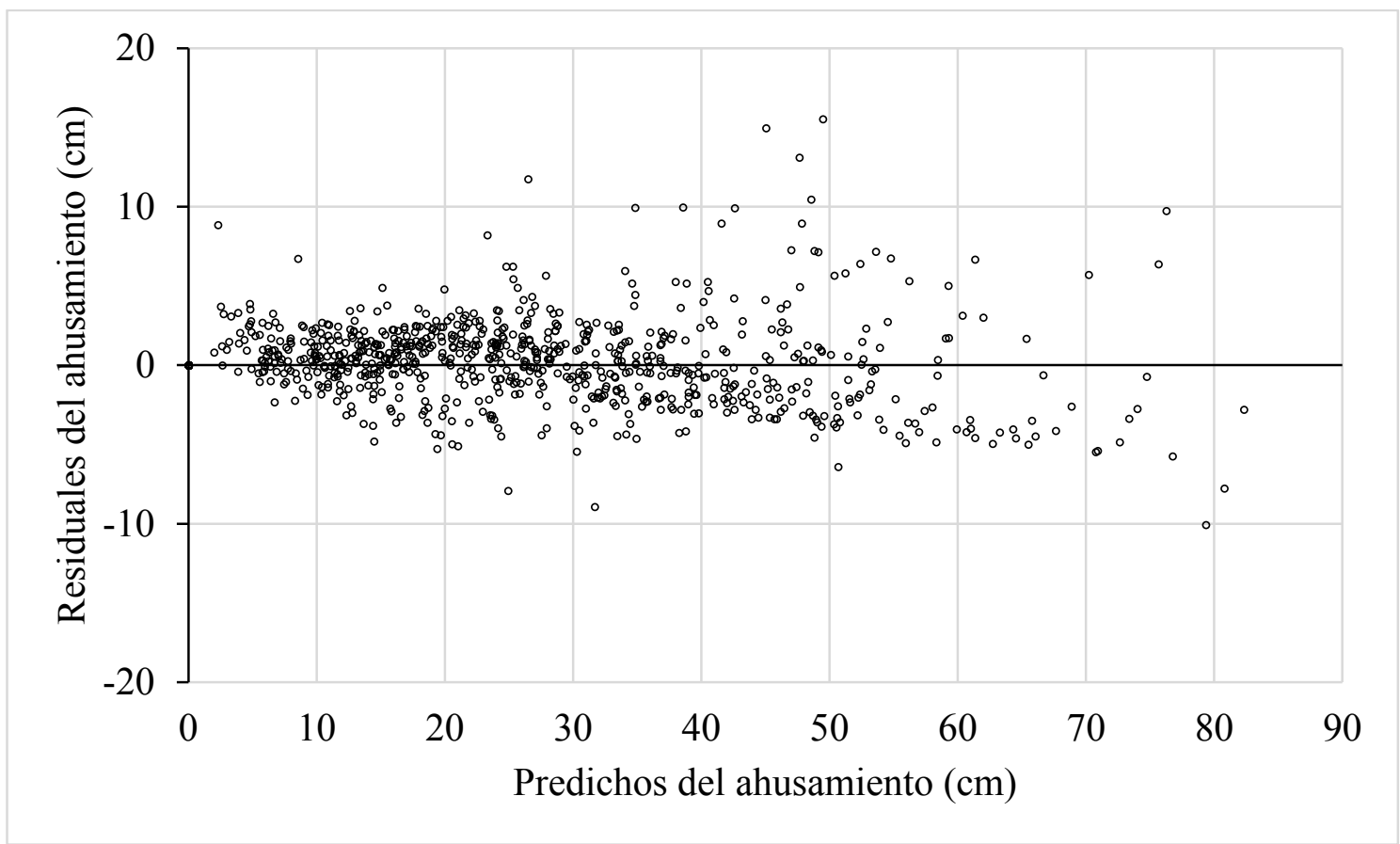

FIGURA 3. Dispersión de residuos frente a los valores estimados del ahusamiento $(\mathrm{dm})$ de Cielito 2.

En la figura 4 se muestra el comportamiento del modelo al seleccionar a un árbol de la categoría diamétrica más representativa de la muestra empleada, que en este caso fue de 40 $\mathrm{cm}$ y altura de $24.2 \mathrm{~m}$.

\section{Verificación de los supuestos del modelo de regresión}

En la tabla 7 se presentan los resultados de las pruebas para los supuestos de homocedasticidad, autocorrelación y normalidad de los residuales. El estadístico de White indicó que las varianzas son heterogéneas, la prueba de Durbin-Watson concluyó que existe autocorrelación positiva en los residuales y la prueba de ShapiroWilk reveló que los residuales no siguen una distribución normal.

\section{Comparación de estimaciones de volumen}

En la tabla 8 se presentan los volúmenes de fuste total para los 13 árboles seleccionados. Las estimaciones realizadas con el modelo de Shumacher-Hall son semejantes al volumen observado y presentan un sesgo en valor absoluto de $0.026 \mathrm{~m}^{3}$; asimismo, el modelo de Berkhout de una entrada mostró un sesgo de 0.054 $\mathrm{m}^{3}$, lo cual indica que posee un sesgo mayor al utilizar solo al diámetro normal como variable independiente. En la estimación de volumen con la integración del modelo Cielito 2, el sesgo absoluto fue de $0.153 \mathrm{~m} 3$ siendo este indicador mayor con respecto a las otras formas de estimación.

\section{DISCUSIÓN}

\section{Modelos de ahusamiento}

El modelo Cielito 1 (Modelo 1) tiende a subestimar los diámetros en la base y a sobreestimar cerca de la punta, de la misma forma el modelo Cielito 3 (Modelo 3) y el modelo de Clutter (1980) (Modelo 4). Los modelos Cielito 2 (Modelo 2) y Biging (1984) (Modelo 5) se ubican en el centro de la distribución de los diámetros $(d m / D)$ de la muestra; ambos describen de forma adecuada las curvas de los perfiles diamétricos; aunque presentan mayor sesgo al estimar los diámetros en la base de los árboles, tienen un comportamiento lógico para el resto del fuste (Fig. 2). 


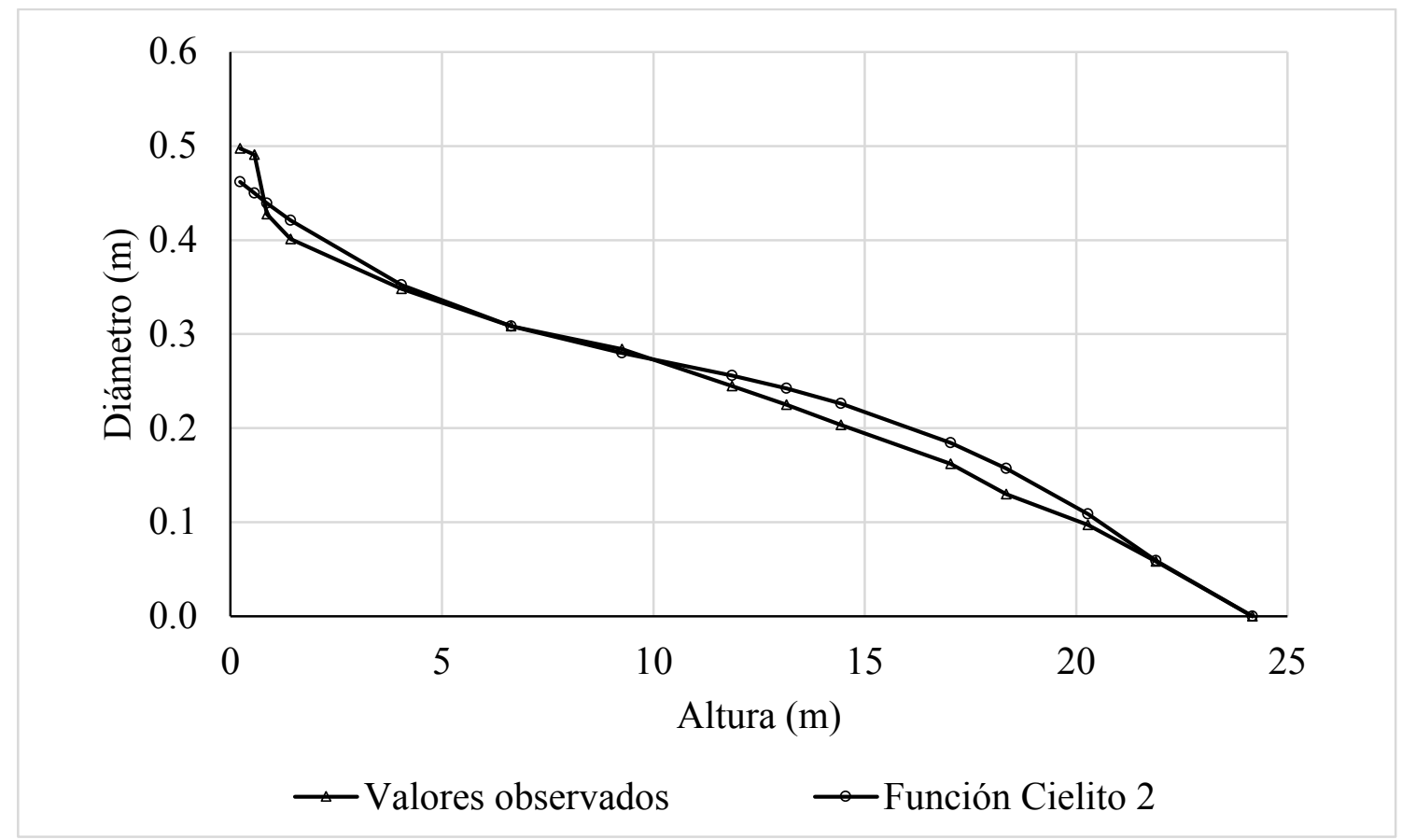

FIgURA 4. Comportamiento de la función de ahusamiento Cielito 2 (sobrepuesta al perfil fustal de P. ayacabuite en la categoría diamétrica de $40 \mathrm{~cm}$.

TABLA 7. Estadísticos de las pruebas de White para heterocedasticidad, de Durbin-Watson para autocorrelación y de Shapiro-Wilk para normalidad de los residuales.

\begin{tabular}{|c|c|c|c|c|c|c|}
\hline \multirow{2}{*}{ Modelo } & \multicolumn{3}{|c|}{ Prueba de White } & \multirow{2}{*}{$\begin{array}{c}\text { Prueba de Durbin- } \\
\text { Watson (DW) }\end{array}$} & \multicolumn{2}{|c|}{ Prueba de Shapiro-Wilk (Wc) } \\
\hline & $n R^{2}$ & $G L$ & $\operatorname{Pr}>x^{2}$ & & $W c$ & $\mathrm{Pr}<W c$ \\
\hline Cielito 2 & 92.78 & 5 & 0.0001 & 0.9548 & 0.95 & 0.0001 \\
\hline
\end{tabular}

$G L$ : grados de libertad; $n R^{2}$ : estadístico de la prueba de White; DW: estadístico de la prueba de Durbin-Watson; Wc: estadístico de la prueba de Shapiro-Willk.

El modelo de ahusamiento Cielito 2 presentó mayor error en la estimación de diámetros en la base y tiende a sobreestimarlo ligeramente en alturas de $12 \mathrm{~m}$ a $20 \mathrm{~m}$ (Fig. 4). Este comportamiento es típico de modelos de ahusamiento generales para árbol individual (modelos polinómicos simples). Bi (2000) y Heidarsson y Pukkala (2011) mencionan que estos enfoques presentan como debilidad sesgos importantes en la estimación de diámetros a distintas alturas, especialmente en la base y en la parte superior del fuste. Por su parte, Newnham (1988) indicó que no existe una teoría que explique adecuadamente la variabilidad de la forma del fuste para todos los árboles. A pesar de estos inconvenientes, la mayor utilidad de los modelos de ahusamiento es para estimar volúmenes comerciales y totales de árboles en pie.
Autores como Hernández-Pérez et al. (2013) y UrangaValencia, De los Santos-Posadas, Valdez-Lazalde, López-Upton y Navarro-Garza (2015) han demostrado que, en rodales naturales y plantaciones con manejo intensivo, los fustes de los árboles presentan una forma más cilíndrica respecto a rodales con manejo extensivo. Por tanto, evaluar el grado de ahusamiento a nivel de especie y localidad de estudio, es importante para determinar con precisión mayor la distribución de productos maderables, dado que el ambiente, la naturaleza genética del individuo y las prácticas silviculturales determinan la forma de los fustes. 
TABLA 8. Comparación del volumen fuste total estimado mediante diferentes estrategias matemáticas.

\begin{tabular}{lllllll}
\hline \multirow{2}{*}{ Árbol } & $D$ & $H$ & \multicolumn{3}{l}{ Volumen total $\left(\mathrm{m}^{3}\right)$} & \\
\cline { 5 - 7 } & $(\mathrm{cm})$ & $(\mathrm{m})$ & Observado & Schumacher-Hall & Berkhout & $\mathrm{C2I}$ \\
\hline 1 & 9.40 & 9.878 & 0.035 & 0.041 & 0.086 & 0.030 \\
2 & 15.60 & 17.350 & 0.189 & 0.179 & 0.237 & 0.146 \\
3 & 20.25 & 20.160 & 0.294 & 0.331 & 0.400 & 0.286 \\
4 & 24.50 & 16.549 & 0.386 & 0.381 & 0.585 & 0.343 \\
5 & 30.00 & 26.910 & 0.929 & 0.890 & 0.876 & 0.837 \\
6 & 35.00 & 29.600 & 1.259 & 1.289 & 1.192 & 1.255 \\
7 & 40.10 & 24.169 & 1.248 & 1.340 & 1.564 & 1.344 \\
8 & 45.40 & 29.244 & 2.096 & 2.024 & 2.004 & 2.085 \\
9 & 51.00 & 31.668 & 2.673 & 2.697 & 2.528 & 2.849 \\
10 & 53.45 & 33.370 & 3.075 & 3.091 & 2.777 & 3.298 \\
11 & 60.50 & 30.110 & 3.221 & 3.476 & 3.556 & 3.813 \\
12 & 65.30 & 30.200 & 3.922 & 3.994 & 4.142 & 4.445 \\
13 & 71.00 & 30.900 & 4.808 & 4.744 & 4.896 & 5.389 \\
& Total & & 24.134 & 24.476 & 24.842 & 26.129 \\
& Promedio & & 1.856 & 1.883 & 1.911 & 2.009 \\
& Desviación estándar & 1.564 & 1.579 & 1.571 & 1.792 \\
& Sesgo & & --- & -0.026 & -0.054 & -0.153 \\
\hline
\end{tabular}

Observado: se cubicó con la fórmula de Smalian y del cono; C2 I: integración de la función de ahusamiento Cielito 2; D: diámetro normal (cm); H: Altura total (m).

\section{Supuestos del modelo de regresión}

El cumplimiento de los supuestos básicos del análisis de regresión no se observó para los residuales del modelo seleccionado (Cielito 2). En el caso del supuesto de normalidad de los residuales solo es necesario cuando se desea establecer intervalos de confianza para los valores medios estimados por el modelo de regresión: un modelo de tendencia central; asimismo, el no cumplimiento de este supuesto no afecta a las predicciones del modelo, siempre y cuando se asegure una extensión suficiente de los valores de la variable dependiente (Aguirre, 1994), como sucede en el presente estudio. La autocorrelación es debida a que la descripción del ahusamiento de los árboles requiere de la obtención de mediciones de los diámetros a diferentes alturas en un mismo individuo, por lo que las observaciones del diámetro están espacialmente correlacionadas (Honorato, 2011). No obstante, es posible minimizar la autocorrelación, mediante la adición de efectos aleatorios con modelos de efectos mixtos (Cruz-Cobos,
De los Santos-Posadas y Valdéz-Lazalde, 2008; QuiñonezBarraza, De los Santos-Posadas, Álvarez-González y VelázquezMartínez, 2014), o al incorporar estructuras de error autorregresivas en la estimación de los parámetros (PompaGarcía, Vega-Muñoz, Soto-Gutiérrez, Trincado y Cruz-Cobos, 2012; López et al., 2015), lo cual permite modelar adecuadamente la autocorrelación de los residuales. Algunos investigadores, entre ellos, Garber y Maguire (2003) emplearon estructuras de error autorregresivas $\operatorname{CAR}(1)$ para modelar el ahusamiento de Pinus ponderosa, P. contorta y Abies grandis en la parte central de Oregón, Estados Unidos; Rojo, Perales, Sánchez, Álvarez y Gadow (2005) emplearon CAR(2) al modelar el ahusamiento de $P$. pinaster en Galicia, España, y López et al. (2015) emplearon CAR(3) para plantaciones de Eucalyptus tereticomis en Colombia.

Kozak (1997) y Rojo et al., (2005) notaron que la corrección de la autocorrelación no mejora de manera importante la capacidad predictiva de los modelos de ahusamiento, porque los 
estimadores de los parámetros y los valores predichos permanecen insesgados en presencia de autocorrelación. Por su parte, Garber y Maguire (2003) y Pompa-García et al. (2012) indican que corregir la autocorrelación tiene sentido porque optimiza la eficiencia en la estimación de parámetros (varianza mínima y errores estándar más reducidos) mejora la interpretación teórica de las propiedades estadísticas de los modelos e identifica las variables predictoras que son significativas.

Por otra parte, Prodan, Peters, Coxy y Real (1997) señalan que la varianza no constante es un fenómeno común al relacionar variables biológicas, lo cual no invalida el ajuste. Los árboles son entes vivientes y su crecimiento es resultado de la actividad de los meristemos primarios, que provoca el crecimiento longitudinal en altura, longitud de las ramas y raíces, así como de los meristemos secundarios, que dan lugar al crecimiento en grosor o en diámetro, al producir nueva madera hacia el interior y corteza hacia el exterior (Diéguez-Aranda et al., 2003). Asimismo, existe una serie de factores edáficos que influyen sobre esta variable, como textura, estructura, profundidad, composición química, $\mathrm{pH}$, aireación, temperatura, capacidad de retención de humedad, conductividad hidráulica, y de la atmosfera, la distribución y cantidad de precipitación, la energía radiante y otros; por tanto, la respuesta fisiológica de los árboles ante estos factores no es homogénea (Lira, 1994); en consecuencia, es de esperarse la presencia de heterocedasticidad (varianza no constante en las perturbaciones del modelo), que no invalida los estimadores de mínimos cuadrados que siguen siendo insesgados, solo que dejan de tener una varianza mínima.

Al no afectar la capacidad de predicción de los modelos de ahusamiento el no cumplimiento de los supuestos subyacentes del análisis de regresión, y sin perder de vista el uso práctico de estos modelos como una herramienta sencilla para modelar el ahusamiento y el volumen fuste total de $P$. ayacahuite, se optó por utilizar el ajuste original (sin corrección por autocorrelación y heterocedasticidad).

\section{Comparación de estimaciones de volumen}

Las estimaciones de volumen con el modelo de doble entrada de Schumacher-Hall son semejantes al volumen observado, con un sesgo bajo. Este modelo ha sido empleado por diversos investigadores por la simplicidad de su estructura, al obtener resultados satisfactorios. Por ejemplo, Pece, Gillard, Galindez y Ríos (2002) emplearon este modelo para la construcción de una tabla de volumen de doble entrada para álamos. Asimismo, Tapia y Navar (2011) utilizaron este modelo para predecir el volumen de Pinus pseudostrobus Lindl., de una fracción de la Sierra Madre Oriental de Nuevo León, México. En tanto que Ramos-Uvilla et al. (2014) emplearon dicho modelo para elaborar tablas de volumen para Pinus lawsonii y Pinus oocarpa en el estado de Michoacán. Por otra parte, las predicciones de volumen realizadas con el modelo de Berkhout de una entrada fueron buenas, con un sesgo de $0.054 \mathrm{~m}^{3}$. En un estudio de volumen para Pinus douglasiana y Pinus oocarpa en el estado de Durango, ValenciaCanales (2013) encontró que el modelo de Berkhout presentó la mayor bondad de ajuste de entre seis modelos de una entrada evaluados, pero su ajuste fue ligeramente menor al compararlo con el modelo de doble entrada de Schumacher-Hall. Sin embargo, al considerar que el modelo de Berkhout utiliza solo al diámetro normal como variable independiente, sus estimaciones son consideradas adecuadas, aunque el uso dependerá de la precisión requerida en la estimación del volumen total.

El modelo Cielito 2 tiene como ventaja ser una función de ahusamiento compatible con una ecuación de volumen, que al ser integrada genera estimaciones de volumen comercial y total similares a las obtenidas por una expresión de volumen existente (Avery y Burkhart, 2001). De esta forma, la ecuación de volumen fuste total generada en este estudio presentó una sobreestimación en el volumen a partir de la categoría de $50 \mathrm{~cm}$, lo que posibilita la utilización preferentemente en categorías menores para determinar la distribución de los productos forestales maderables de P. ayacabuite.

El modelo de ahusamiento Cielito 2 con la respectiva ecuación de volumen de fuste total, complementa el sistema volumétrico para $P$. ayacahuite de los bosques de Ixtlán de Juárez, Oaxaca. Este sistema está integrado por modelos de volumen fuste total de una y dos entradas (Ramírez-Martínez et al., 2016a) y por un sistema segmentado de ahusamiento-volumen compatible (Ramírez-Martínez, Santiago-García, QuiñonezBarraza, Ruiz-Aquino y Rodríguez-Ortiz, 2016b) para la estimación del volumen total y comercial variable, así como la distribución de productos de manera más precisa. Sin embargo, 
los modelos aquí presentados representan un enfoque más sencillo de implementar en la práctica, y generan estimaciones adecuadas del volumen y la distribución de productos de árboles en pie.

\section{CONCLUSIONES}

Entre los cinco modelos evaluados para describir el ahusamiento de árboles de Pinus ayacabuite Ehren, tres de estos (Cielito 2, Cielito 3 y Biging, 1984) presentaron la mejor calificación en términos de los estadísticos de bondad de ajuste. El modelo Cielito 2 obtuvo la mejor calificación, seguido por el modelo de Biging (1984) y Cielito 3; en el análisis gráfico de la estimación del perfil diamétrico del árbol se observó que estos modelos tienen un comportamiento similar, presentan capacidad baja para estimar diámetros en la base del fuste y tienden ligeramente a una sobreestimación en la parte media. A pesar de lo anterior, la capacidad predictiva se considera adecuada para fines de distribución de productos a lo largo del fuste; aunado a esto, los indicadores de ajuste sugieren un modelado robusto. En este estudio se optó por el modelo Cielito 2 debido a que presentó un comportamiento más consistente.

De las distintas formas para realizar estimaciones de volumen, la más adecuada resultó el modelo de doble entrada, por presentar un sesgo menor y ser de fácil aplicación; aunque la integración del modelo Cielito 2 posee la capacidad de estimar el volumen entre dos alturas a lo largo del fuste, su utilidad mayor es para realizar la desagregación de productos maderables de árboles en pie.

\section{REFERENCIAS}

Aguirre, J. A. (1994). Introducción al tratamiento de series temporales (1a ed.). Madrid, España: Ediciones Díaz de Santos S. A.

Avery, T. E., \& Burkhart, H. E. (2001). Forest Measurements (5a ed). Nueva York, Estados Unidos: McGraw-Hill.

Bi, H. (2000). Trigonometric variable-form taper equations for Australian Eucalyptus. Forest Science, 46(3), 397-407.

Biging, G. S. (1984). A compatible volume- taper function for Alberta trees. Forest Science, 14, 339-350.

Bruce, R., Curtis, L., \& Van Coevering, C. (1968). Development of a system of taper and volume tables for red alder. Forest Science, 14(3), 339-350.
Burkhart, H. E., \& Tome, M. (2012). Modeling forest trees and stands. Nueva York, Estados Unidos: Springer. doi: 10.1007/978-90-481-31709

Cao, Q. V., Burkhart, H. E., \& Max, T. A. (1980). Evaluation of two methods for cubic-volume prediction of loblolly pine to any merchantable limit. Forest Science, 26(1), 71-80.

Castellanos-Bolaños, J. F., Treviño-Garza, E. J., Aguirre-Calderón, O. A., Jiménez-Pérez, J., \& Velázquez-Martínez, A. (2010). Diversidad arbórea y estructura espacial de bosques de pinoencino en Ixtlán de Juárez, Oaxaca. Revista Mexicana de Ciencias Forestales, 1(2), 39-52.

Clutter, J. L. (1980). Development of taper function from variable top merchantable volume equations. Forest Science, 26, 117-120.

Cruz-Cobos, F., De los Santos-Posadas H. M., \& Valdéz-Lazalde, J. R. (2008). Sistema compatible de ahusamiento-volumen para Pinus cooperi Blanco en Durango México. Agrociencia, 42, 473-485.

Davel, M., \& Trincado, G. (2000). Evaluación de modelos fustales para Pseudotsuga menziesii en la Patagonia Andina Argentina. Investigación Agraria: Sistemas y Recursos Forestales, 9(1), 103-116.

Demaerschalk, J. P. (1972). Converting volume equations to compatible taper equations. Forest Science, 18(3), 241-245.

Díaz F., M. M., \& Llorente M., M. M. (2013). Econometría (4a ed.). Madrid, España: Pirámide.

Di Rienzo, J. A., Casanoves, F., González, L. A., Tablada, E. M., Díaz, M. P., Robledo, C. W., \& Balzarini, M. G. (2005). Estadística para las ciencias agropecuarias (6a ed.). Córdoba, Argentina: Brujas.

Diéguez-Aranda, U., Barrio-Anta, M., Castedo-Dorado, F., RuízGonzález, A. D., Alvaréz-Taboada, M. F., Alvaréz-González, J. G., \& Rojo-Alboreca, A. (2003). Dendrometría. Madrid, España: Mundi-Prensa. doi: 10.1139/x06-008

Durbin, J., \& Watson, G. S. (1971). Testing for serial correlation in least squares regression III. Biometrika, 58, 1-19. doi: 10.2307/2334313

Fang, Z., \& Bailey, R. L. (1999). Compatible volume and taper models with coefficients for tropical species on Hainan Island in southern China. Forest Science, 45, 85-100.

Garber, S. M., \& Maguire, D. A. (2003). Modeling stem taper of three central Oregon species using nonlinear mixed effects models and autoregressive error structures. Forest Ecology and Management, 179, 507-522. doi: 10.1016/S0378-1127(02)00528-5

Heidarsson, L., \& Pukkala, T. (2011). Taper functions for lodgepole pine (Pinus contorta) and siberian larch (Larix sibirica) in Iceland. Icelandic Agricultural Sciences, 24, 3-11.

Hernández-Pérez, D., De los Santos-Posadas, H. M., Ángeles-Pérez, G., Valdéz-Lazalde, J. R., \& Volke-Haller, V. H. (2013). Funciones de ahusamiento y volumen comercial para Pinus patula Schltdl. et Cham. en Zacualtipán, Hidalgo. Revista Mexicana de Ciencias Forestales, 4(16), 34-45. 
Honorato S., J. A. (2011). Modelos volumétricos fustales para Acrocarpus fraxinifolius Wight \& Arn. en plantaciones agroforestales de la sierra norte de Puebla, México. Revista Mexicana de Ciencias Forestales, 2(6), 55-71.

Kozak, A. (1997). Effects of multicollinearity and autocorrelation on the variable-exponent taper functions. Canadian Journal of Forest Research, 27, 619-629. doi: 10.1139/x97-011

Kozak, A. (2004). My last words on taper equations. The Forestry Chronicle, 80(4), 507-515. doi: $10.5558 / \mathrm{tfc} 80507-4$

Lee, W. K., Seo, H. J., Son, M. Y., Lee, H. K., \& Gadow, K. V. (2003). Modeling stem profiles for Pinus densiflora in Korea. Forest Ecology and Management, 172(1), 69-77. doi: 10.1016/S03781127(02)00139-1

Lira S., R. H. (1994). Fisiología vegetal. México: Trillas.

López, M. A., Barrios, A., \& Trincado, G. (2015). Modelos de perfiles fustales con una estructura de error autorregresiva para plantaciones de Eucalyptus tereticornis en Colombia. Madera $y$ Bosques, 21(2), 73-88. doi: 10.21829/myb.2015.212446

Max, T. A., \& Burkhart, H. E. (1976). Segmented polynomial regression applied to taper equations. Forest Science, 22(3), 283-289.

Newnham, R. (1988). A variable-form taper function (Report PI-X-083). Chalk River, Ontario, Canadá: Petawawa National Forestry Institute.

Omerod, D. W. (1973). A simple bole model. The Forestry Chronicle, 49, 136-138. doi: 10.5558/tfc49136-3

Parresol, B. R., \& Thomas, C. C. (1996). A simultaneous density-integral system for estimating stem profile and biomass: Slash pine and Willow Oak. Canadian Journal of Forest Research, 26, 773-781.

Pece, M., Gillard, C., Galindez, M., \& Ríos, N. (2002). Tabla de volumen de doble entrada para álamos de la zona de riego de Santiago del Estero, Argentina. Quebracho, 9(3), 95-105.

Pérez, D. N., Burkhart, H. E., \& Stiff, C. T. (1990). A variable-form taper function for Pinus oocarpa Schiede in Central Honduras. Forest Science, 36(1), 186-191.

Pompa-García, M., Corral-Rivas, J. J., Hernández-Díaz, J. C., \& AlvarezGonzález J. G. (2009a). A system for calculating the merchantable volume of oak trees in the northwest of the state of Chihuahua, Mexico. Journal of Forestry Research, 20(4), 293-300. doi: 10.1007/s11676-009-0051-x

Pompa-García, M., Hernández, C., Prieto-Ruiz, J. A., \& Dávalos-Sotelo, R. (2009b). Modelación del perfil fustal de Pinus durangensis en Guachochi, Chihuahua, México. Madera y Bosques, 15(1), 61-73. doi: $10.21829 /$ myb.2009.1511197

Pompa-García, M., Vega-Muñoz, J., Soto-Gutiérrez, R., Trincado, G., \& Cruz-Cobos, F. (2012). Estimates of the bark thickness in bole profiles of oak in northern Mexico. Research Journal of Forestry, 6 (2), 32-40. doi: 10.3923/rjf.2012.32.40
Prodan, M., Peters, R., Coxy, F., \& Real, P. (1997). Mensura Forestal. Serie Investigación y Educación en Desarrollo Sostenible Proyecto IICA/GTZ. San José, Costa Rica.

Quiñonez-Barraza, G., De los Santos-Posadas, H. M., ÁlvarezGonzález, J. G., \& Velázquez-Martínez, A. (2014). Sistema compatible de ahusamiento y volumen comercial para las principales especies de Pinus en Durango, México. Agrociencia, 48(5), 553-567.

Ramírez-Martínez, A., Santiago-García, W., Quiñonez-Barraza, G., RuizAquino, F., \& Martínez-Antúnez, P. (2016a). Modelos de volumen fustal para Pinus ayacabuite Ehren. Revista Mexicana de Agroecosistemas, 3(2), 61-74.

Ramírez-Martínez, A., Santiago-García, W., Quiñonez-Barraza, G., RuizAquino, F., \& Rodríguez-Ortiz, G. (2016b). Sistema compatible segmentado de ahusamiento-volumen comercial para Pinus ayacabuite Ehren. En F. Gallardo-López (Ed.), Innovando el Agro Veracruzano 2016. Frente a los retos de la relación Sociedad-Naturaleza (pp. 639-659). Veracruz, México: Colegio de Postgraduados.

Ramos-Uvilla, J. A., García-Magaña, J. J., Hernández-Ramos, J., GarcíaCuevas, X., Velarde-Ramírez, J. C., Muñoz-Flores, H. J., \& García E., G. G. (2014). Ecuaciones y tablas de volumen para dos especies de Pinus de la Sierra Purépecha, Michoacán. Revista Mexicana de Ciencias Forestales, 5(23), 92-109.

Real, P. L., \& Moore, J. A. (1986). An individual tree system for Douglasfir in the inland north-west. En A. R. Ek, S. R. Shifley, \& T. E. Burk. Forest growth modelling and prediction (p. 1037-1044). Proceeding of IUFRO Conference, 24-28 August 1986. Minneapolis, Minnesota, EUA.

Rentería, A. J. B., Ramírez, M. H., \& Zamudio, S. F. (2006). Sistema de cubicación para Pinus cooperi Blanco mediante ecuaciones de ahusamiento en San Dimas, Durango. En CEVAG. El Sitio Permanente de Experimentación Forestal (SPEF) "Cielito Azul" a 40 años de su establecimiento (p. 135). Publicación Especial Núm. 23. San Dimas, México: Instituto Nacional de Investigaciones Forestales, Agrícolas y Pecuarias.

Rojo, A., Perales, X., Sánchez, F., Álvarez, J., \& Gadow, K. V. (2005). Stem taper functions for maritime pine (Pinus pinaster Ait.) in Galicia (northwestern Spain). European Journal of Forest Research, 124, 177-186. doi: 10.1007/s10342-005-0066-6

Ruiz-Aquino, F., Valdez-Hernández, J. I., Manzano-Méndez, F., Rodríguez-Ortiz, G., Romero-Manzanares, A., \& Fuentes-López, M.E. (2014). Ecuaciones de biomasa aérea para Quercus laurina y Q. crassifolia en Oaxaca, México. Madera y Bosques, 20(2), 33-48. doi: 10.21829/myb.2014.202162

SAS Institute Inc. (2011). SAS/ETS* 9.0 User's guide. Carry. Cary, NC. SAS Institute Inc.

Shapiro, S., \& Wilk, M. (1965). An analysis of variance test for normality. Biometrika Trust, 52(3/4), 591-611. doi: 10.2307/2333709 
Tapia, J., \& Navar, J. (2011). Ajuste de modelos de volumen y funciones de ahusamiento para Pinus pseudostrobus Lindl. en bosques de pino de la Sierra Madre Oriental de Nuevo León, México. Revista Forestal Veracruzana, 13(2), 19-28.

Uranga-Valencia, L. P., De los Santos-Posadas, H. M., Valdez-Lazalde, J. R., López-Upton, J., \& Navarro-Garza, H. (2015). Volumen total y ahusamiento para Pinus patula Schiede ex Schltdl. et Cham. en tres condiciones de bosque. Agrociencia, 49(7), 787-801.

Valencia-Canales, J. E. (2013). Sistema volumétrico para Pinus oocarpa Schiede y Pinus douglasiana Martínez en el estado de Durango (tesis de maestría no publicada). Universidad Autónoma de Nuevo León, México.

White, H. (1980). A heteroskedasticity-consistent covariance matrix and direct test for heteroskedasticity. Econometrica, 48(4), 817-838. doi: $10.2307 / 1912934$
Manuscrito recibido el: 18 de enero de 2017

Aceptado el: 12 de septiembre de 2017

Publicado el: 30 de mayo de 2018

Este documento se debe citar como:

Ramírez-Martínez, A., Santiago-García, W., Quiñonez-Barraza, G., RuizAquino, F., \& Antúnez, P. (2018). Modelación del perfil fustal y volumen total para Pinus ayacahuite Ehren. Madera y Bosques, 24(2), e2421496. doi:10.21829/myb.2018.2421496

Madera y Bosques por Instituto de Ecología, A.C. se distribuye bajo una Licencia Creative Commons Atribución-NoComercialCompartirlgual 4.0 Internacional. 
ANEXO 1. Integración del modelo de ahusamiento Cielito 2.

La integración del modelo de ahusamiento Cielito 2 (Rentería et al., 2006) para obtener una expresión de volumen fuste total se realizó considerando $h_{1}=0$ y $h_{2}=H$.

$$
V=K \int_{h_{1}}^{h_{2}} D^{2}\left(\beta_{0}+\beta_{1} X+\beta_{2} X^{2}+\beta_{3} X^{3}+\beta_{4} X^{4}\right) d X
$$

Donde:

$$
\begin{aligned}
& K=\frac{\pi}{40000} \\
& X=\left(\frac{h m}{H}\right) \\
& V=K D^{2} \int_{h_{1}}^{h_{2}}\left[\beta_{0}+\beta_{1}\left(\frac{h m}{H}\right)+\beta_{2}\left(\frac{h m}{H}\right)^{2}+\beta_{3}\left(\frac{h m}{H}\right)^{3}+\beta_{4}\left(\frac{h m}{H}\right)^{4}\right) d X \\
& V=K D^{2}\left[\beta_{0}(h m)+\beta_{1} \frac{(h m)^{2}}{2 H}+\beta_{2} \frac{(h m)^{3}}{3 H^{2}}+\beta_{3} \frac{(h m)^{4}}{4 H^{3}}+\beta_{4} \frac{(h m)^{5}}{5 H^{4}}\right]_{h_{1}}^{h_{2}}
\end{aligned}
$$

Cuando $h m=H$, se obtiene la ecuación de volumen fuste total:

$$
\begin{aligned}
& V=K D^{2}\left[\beta_{0}(H)+\beta_{1} \frac{(H)^{2}}{2 H}+\beta_{2} \frac{(H)^{3}}{3 H^{2}}+\beta_{3} \frac{(H)^{4}}{4 H^{3}}+\beta_{4} \frac{(H)^{5}}{5 H^{4}}\right] \\
& V=K D^{2}\left[\beta_{0}(H)+\beta_{1} \frac{H}{2}+\beta_{2} \frac{H}{3}+\beta_{3} \frac{H}{4}+\beta_{4} \frac{H}{5}\right] \\
& V=K D^{2} H\left[\beta_{0}+\frac{\beta_{1}}{2}+\frac{\beta_{2}}{3}+\frac{\beta_{3}}{4}+\frac{\beta_{4}}{5}\right]
\end{aligned}
$$

